\title{
Anisotropic Complex Refractive Indices of Atomically Thin Materials: Determination of the Optical Constants of Few-Layer Black Phosphorus
}

\author{
Aaron M. Ross ${ }^{1}$, Giuseppe M. Paternò ${ }^{2}\left(\mathbb{D}\right.$, Stefano Dal Conte ${ }^{1}$, Francesco Scotognella ${ }^{1,2, *(\mathbb{C})}$ \\ and Eugenio Cinquanta ${ }^{3}$ (I) \\ 1 Dipartimento di Fisica, Politecnico di Milano, Piazza Leonardo da Vinci 32, 20133 Milan, Italy; \\ aaronmichael.ross@polimi.it (A.M.R.); stefano.dalconte@polimi.it (S.D.C.) \\ 2 Center for Nano Science and Technology@PoliMi, Istituto Italiano di Tecnologia (IIT), Via Giovanni Pascoli, \\ 70/3, 20133 Milan, Italy; Giuseppe.Paterno@iit.it \\ 3 Istituto di Fotonica e Nanotecnologie, Consiglio Nazionale delle Ricerche, 20133 Milan, Italy; \\ eugenioluigi.cinquanta@cnr.it \\ * Correspondence: francesco.scotognella@polimi.it; Tel.: +39-02-2399-6056
}

Received: 5 November 2020; Accepted: 14 December 2020; Published: 16 December 2020

\begin{abstract}
In this work, studies of the optical constants of monolayer transition metal dichalcogenides and few-layer black phosphorus are briefly reviewed, with particular emphasis on the complex dielectric function and refractive index. Specifically, an estimate of the complex index of refraction of phosphorene and few-layer black phosphorus is given. The complex index of refraction of this material was extracted from differential reflectance data reported in the literature by employing a constrained Kramers-Kronig analysis combined with the transfer matrix method. The reflectance contrast of 1-3 layers of black phosphorus on a silicon dioxide/silicon substrate was then calculated using the extracted complex indices of refraction.
\end{abstract}

Keywords: transition metal dichalcogenides; black phosphorus; two-dimensional materials; complex refractive index; Kramers-Kronig analysis

\section{Introduction}

Two-dimensional materials emerged as a promising option for the development of nanoelectronic and optoelectronic devices, due to their peculiar electronic and optical properties. In this respect, transition metal dichalcogenides (TMDs), when thinned to a single atomic layer, show an indirect-direct band gap transition, inequivalent valleys in the Brillouin zone and non-trivial topological order [1-3]. In the vast family of two-dimensional materials, elemental ones represent a niche, due to their high reactivity at ambient conditions, that makes their characterization and exploitation challenging $[4,5]$. Among them, phosphorene, i.e., monolayer black phosphorous (BP), is the most promising material for optoelectronic and photonic applications, as its electronic band gap lies in a spectral region between the ones of TMDs and the one of graphene [6]. For an effective employment of these materials in photonic and optoelectronic devices, it is important to determine their dielectric response.

Among the monolayers of transition metal dichalcogenides, $\mathrm{MoS}_{2}$ was the first material on which optical measurements were performed. In fact, in 2013 and 2014, the complex dielectric function of $\mathrm{MoS}_{2}$ was measured with spectroscopic ellipsometry by Shen et al. [7], Yim et al. [8] and Li et al. [9]. The complex dielectric functions of monolayers $\mathrm{MoS}_{2}, \mathrm{WS}_{2}, \mathrm{MoSe}_{2}$ and $\mathrm{WSe}_{2}$ were measured by $\mathrm{Li}$ et al. by employing reflectance contrast spectroscopy [10]. In 2019, the monolayers, together with the bilayers and the trilayers, of the four above mentioned chalcogenides were studied by Hsu et al. [11]. In 2020, Ermolaev et al. [12] measured the complex dielectric function of $\mathrm{MoS}_{2}$ in a broad range of 
wavelengths, i.e., $290-3300 \mathrm{~nm}$. Ermolaev et al. [13] also measured the complex index of refraction of $\mathrm{WS}_{2}$ in the range of $375-1700 \mathrm{~nm}$.

Additionally, a number of studies investigated the anisotropic components of TMD and phosphorous few-layer systems via the incorporation of dielectric and plasmonic nanostructures [14-21]. In one study, the coupling of electromagnetic fields with the out-of-plane component of the $\mathrm{MoS}_{2}$ dielectric function was possible due to the peculiar morphology of chemical vapor deposition (CVD)-grown few-layer $\mathrm{MoS}_{2}$ on rippled substrate, hence promoting 1D nanostructures as a promising path for the full exploitation of the few-layer $\mathrm{MoS}_{2}$ dielectric response [19]. Similarly, a top-down approach was revealed to be effective for the strain-induced modification of the electronic band structure of atomically thin $\mathrm{MoS}_{2}$ and black phosphorous, paving the way for the on-demand tuning of their optical properties $[20,21]$. One study investigated exfoliated $\mathrm{MoS}_{2}$ on a lithographically defined $\mathrm{SiO} 2$ nanocone substrate, demonstrating a deterministic red-shift of the A exciton photoluminescence (PL) due to elastic strain [14]. Another study utilized spatially and time-resolved PL diffusion measurements of $\mathrm{WSe}_{2}$ exfoliated onto a $1.5 \mu \mathrm{m}$ diameter $\mathrm{SiO}_{2}$ pillar, resulting in a similar red-shift and demonstration of exciton diffusion towards high tensile strain regions; that work was a proof of concept for engineerable excitonic diffusion that may lead to a new generation of opto-excitonic devices [15].

Strain engineering may also be used to enable new radiative pathways for the optical excitation of selection rule forbidden TMD dark excitons under normal incidence conditions; these dark and "gray" excitons possess much longer radiative lifetimes (>100 ps) $[16,22,23]$. In the absence of intentional strain, one study utilized a high numerical aperture objective lens $(\mathrm{NA}=0.82)$ to characterize the dark exciton in $\mathrm{WSe}_{2}$ : even for normal incidence, a Gaussian beam with a tight focus has a non-zero electric field component along the beam propagation direction [16]. However, optical excitation of the dark exciton in $\mathrm{WSe}_{2}$ was more easily enabled by coupling to the surface plasmon polariton of silver (Ag) [17]. Another study incorporated a hexagonal boron nitride (hBN)-encapsulated WSe2 monolayer into a plasmonic modulator device, requiring precise knowledge of the TMD, gold $(\mathrm{Au})$ and $\mathrm{hBN}$ complex indices of refraction [18]. It thus emerges how the development of simple and robust tools for the careful analysis of the dispersion of the complex refractive index is beneficial for an appropriate engineering of devices that include two-dimensional materials.

In contrast, phosphorene (single-layer BP) and few-layer BP have not been studied with the same fervor as their TMD counterparts, likely due to their relative sensitivity to degradation. One significant distinction between TMDs and BP is that the band structure of BP exhibits considerable anisotropy: that is, the effective electron mass along the armchair and zig-zag directions is a factor of five times larger in the former direction [24]. This anisotropy is manifest in polarization-sensitive absorption and PL measurements [24-27], as well as spatially resolved excitonic diffusion measurements [24]. The relatively fragile phosphorene system holds considerable promise due to the ease of PL/absorption tunability by layer number tuning, which can shift the resonance energies between 0.32 and $1.7 \mathrm{eV}$ [24].

We focus on estimating the complex refractive index of phosphorene, which shows a morphological in-plane anisotropy resulting in an anisotropic optical response [25]. We retrieve the refractive index along the armchair (AC) and zig-zag (ZZ) direction by exploiting the constrained Kramers-Kronig analysis (CKKA) and fit experimental contrast reflectivity with a model obtained by means of the transfer matrix method (TMM).

\section{Materials and Methods}

Methodology for extraction of the complex index of refraction of few-layer BP from reflectance contrast data: The linear optical properties of thin films are commonly measured via static absorption or reflectivity contrast measurements. Experimental techniques include normal incidence reflectivity contrast (dR/R) over a wide plane of view, spatially resolved confocal $\mathrm{dR} / \mathrm{R}$ in the case of exfoliated small-area TMDs, few-layer BP, graphene and graphene oxide and simultaneous absolute reflectivity and transmission measurements for the determination of static absorption [10,11,25,27-30]. Although these methods reveal the static absorption $A$ and reflectivity $\mathrm{dR} / \mathrm{R}$ and transmission $\mathrm{dT} / \mathrm{T}$ contrasts of the thin films 
relative to the linear substrate response, the determination of the complex index of refraction $\widetilde{n}=n+i k$, or equivalently the complex dielectric function $\varepsilon=\varepsilon_{r}+i \varepsilon_{i}$, where $n=\sqrt{\varepsilon}$, typically requires more sophisticated optical characterization methods such as ellipsometry [8,31-33].

In this section, we extract the complex index of refraction of hBN-encapsulated few-layer BP on a sapphire substrate from confocal reflectivity contrast data taken from [25]. Specifically, the layered sample structure is as follows (see Figure 1): air $\rightarrow 15 \mathrm{~nm} \mathrm{hBN} \rightarrow$ few-layer BP $\rightarrow \alpha-\mathrm{Al}_{2} \mathrm{O}_{3}$ (sapphire); $\mathrm{BP}$ thicknesses range nominally from $0.5 \mathrm{~nm}(1 \mathrm{~L})$ to $2.5 \mathrm{~nm}(5 \mathrm{~L})$, and bulk BP is $100 \mathrm{~nm}$ thick. We assume that the exfoliated hBN layer is crystalline, rather than isotropic as for the case of cubic BN or polycrystalline hBN [33-35] with an in-plane dielectric constant given by

$$
\varepsilon_{h B N, \|}=n_{h B N, \|}^{2}=1+\frac{3.336 \lambda^{2}}{\lambda^{2}-26322}
$$

where the wavelength $\lambda$ is given in nanometers [33]. Additionally, we also use a Sellmeier equation to describe the $\alpha-\mathrm{Al}_{2} \mathrm{O}_{3}$ substrate index of refraction [36].

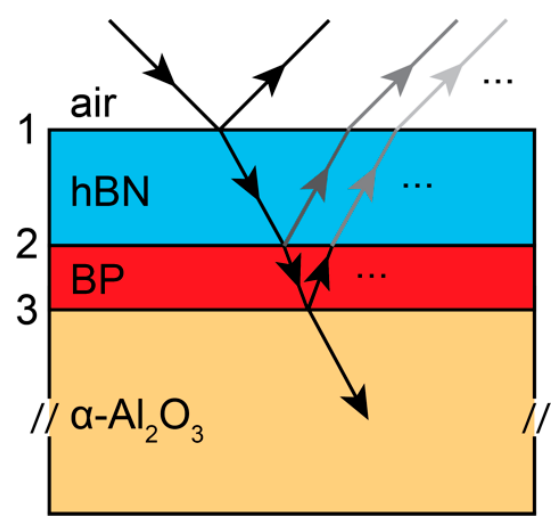

Figure 1. Schematic diagram of multi-layer system under study: air, $15 \mathrm{~nm}$ of hBN, 1-5 layers of black phosphorus (BP) and a thick substrate of $\alpha-\mathrm{Al}_{2} \mathrm{O}_{3}$ [25]. Input and output wave are indicated directionally by arrows, where only a few reflected waves from each interface are indicated. The incident angle is shown as oblique for clarity (normal incidence in experiment).

One commonly used method for the determination of the complex linear material response functions is the Kramers-Kronig analysis (KKA): it is well known that the real and imaginary parts of the dielectric function are related to one another via the Kramers-Kronig (KK) relations, which impose causality conditions on the response functions [37-39]. However, the integrals involved in these relations are taken over infinite limits; practical implementation requires the acquisition of data over a wide energy range, and may be limited to materials which exhibit an optical response only over a narrow wavelength range.

We utilize an alternative method called the constrained Kramers-Kronig analysis (CKKA), which was introduced by Kuzmenko to address some of the problems associated with KKA [37]. This method takes advantage of the fact that basis functions such as the complex Lorentzian can be chosen that individually satisfy the KK relations. Such a function is given by

$$
\varepsilon_{k}=\frac{\omega_{p, k}^{2}}{\omega_{0, k}^{2}-\omega^{2}+i \omega \gamma_{k}}
$$

where $\omega_{p, k}^{2}$ is the plasma frequency, $\omega_{0, k}^{2}$ is the oscillator frequency and $\gamma_{k}$ is the linewidth. Since linear combinations of such basis functions also satisfy the KK relations, the method introduces a mesh in energy space of a large number of oscillators $N_{o s c} \sim N_{\text {data }}$; the oscillator frequencies are fixed, and the linewidth is chosen as the spacing between oscillator energies. Thus, the only remaining parameters 
left to minimize are the oscillator strengths (or plasma frequencies): hence, the constrained KK analysis. To further optimize the CKKA fitting procedure, we chose functions that are similar to the complex Lorentzian of Equation (2) and also satisfy the KK relations, but are more locally weighted, avoiding the contributions of the tails of the Lorentzian distributions far away from the central oscillator energy. One such function is a triangular function for $\varepsilon_{i}$, with a corresponding analytically integrable $\varepsilon_{r}[37,38]$.

We note that although this method can easily reproduce reflectivity contrast data when a sufficiently large oscillator mesh is used, it is sensitive to a lack of information about the optical response outside of the experimental acquisition range $[10,37,38]$. This problem is not entirely unexpected, considering that it is fundamentally a KK-type analysis method. Often, high-frequency information about a given optically active system is known via X-ray photoelectron emission spectroscopy or UV absorption experiments; additionally, low-frequency information is often revealed via Fourier transform IR (FTIR) and Raman scattering experiments [5,40-42]. However, such information is currently lacking for few-layer BP, although bulk BP is well characterized. Although similar calculations using the CKKA method have extracted the complex index of refraction for TMD monolayer and few-layer systems assuming a bulk-like response at high energies [10], no such assumptions are made here for the few-layer BP fitting procedure.

For a given complex index of refraction, the optical response of a multi-layered system is then determined by the transfer matrix method (TMM) [43]. It is noted that perturbative "linearized" methods have also been developed for the treatment of TMD monolayers, few-layer BP and graphene [10,25,30], which treat the optical response of the thin film using the sheet conductivity

$$
\sigma^{d}=\sigma d=-i d \omega(\varepsilon-1) .
$$

For basic layered systems such as air $\rightarrow$ monolayer $\mathrm{TMD} \rightarrow \mathrm{SiO}_{2}$, relatively simple forms for the reflectivity contrast $\mathrm{dR} / \mathrm{R}$ can be derived [29]. However, for the system under study in this section (air $\rightarrow \mathrm{hBN} \rightarrow \mathrm{BP} \rightarrow \alpha-\mathrm{Al}_{2} \mathrm{O}_{3}$ ), with no assumptions being made about reflection coefficients between interfaces, no tractable analytical form for $\mathrm{dR} / \mathrm{R}$ is derived; note that this stands in contrast to previous studies [25] which assume that the $\mathrm{BP} \rightarrow \alpha-\mathrm{Al}_{2} \mathrm{O}_{3}$ interface does not contribute to the overall optical response of the layered system.

We use the standard TMM [43] to relate the input and reflected fields at the air $\rightarrow \mathrm{hBN}$ interface (1) to the transmitted fields at the $\mathrm{BP} \rightarrow \alpha-\mathrm{Al}_{2} \mathrm{O}_{3}$ interface (3). Specifically, for normal incidence fields, the electric and magnetic fields, which are implicit sums of all fields (incident, reflected, transmitted) are given by

$$
\left[\begin{array}{c}
E_{1} \\
H_{1}
\end{array}\right]=\hat{M}_{1-2} \hat{M}_{2-3}\left[\begin{array}{c}
E_{3} \\
H_{3}
\end{array}\right], \hat{M}_{i-j}=\left[\begin{array}{cc}
\cos \phi_{i j} & \frac{i}{n_{i j}} \sin \phi_{i j} \\
i n_{i j} \sin \phi_{i j} & \cos \phi_{i j}
\end{array}\right], \phi_{i j}=\frac{2 \pi n_{0} n_{i j} d_{i j} E}{h c}
$$

which can then be related to the field reflection coefficient by the following relation

$$
r=\frac{E_{r}}{E_{i}}=\frac{n_{0} m_{11}+n_{0} n_{s} m_{12}-m_{21}-n_{s} m_{22}}{n_{0} m_{11}+n_{0} n_{s} m_{12}+m_{21}+n_{s} m_{22}}
$$

where $m_{i j}$ is the $i j$-th element of the product of the two matrices in Equation (4), $n_{0}, n_{s}$ are the indices of refraction of air and the back substrate $\left(\alpha-\mathrm{Al}_{2} \mathrm{O}_{3}\right), h c$ is the product of the speed of light and Planck's constant, $E=\frac{h c}{\lambda}$ is energy, $n_{i j}$ is the index of refraction for the material layer between the $i$ and $j$ interfaces and $d_{i j}$ is the thickness between the $i$ and $j$ interfaces (Figure 1). Finally, the reflectance contrast is given by

$$
\frac{d R}{R}=\left(\left|r_{r e f}\right|^{2}-\left|r_{\text {sample }}\right|^{2}\right) /\left|r_{r e f}\right|^{2}
$$

where $r_{r e f}$ is the field reflection coefficient for the reference air $\rightarrow \mathrm{hBN} \rightarrow \alpha-\mathrm{Al}_{2} \mathrm{O}_{3}$, which is determined by setting $\hat{M}_{2-3}=\hat{1}$. This procedure makes no perturbative assumptions about the index of refraction of $\mathrm{BP}$, and allows for generalization to thicker layers such as the bulk BP case. 


\section{Results and Discussion}

Complex index of refraction for few-layer and bulk BP: The complex index of refraction for few-layer $\mathrm{BP}$ is then determined by fitting the reflectance contrast data [25] using non-linear least squares fitting of the weight (plasma frequencies) parameters in the CKKA method with triangular basis functions; this method is referred to as CKKA+TMM $[37,38]$. The $d R / R$ data were extracted from the literature using a plot digitizer with high accuracy [44]. The resulting $\mathrm{dR} / \mathrm{R}$ fits, real indices of refraction $n$ and extinction coefficients $k$, for both $\mathrm{x}$ - and $\mathrm{y}$-polarized excitation for one to five layers and bulk BP, are displayed in Figure 2. We note the high quality of the $\mathrm{dR} / \mathrm{R}$ fits (Figure 2a-e), even small variations in $\mathrm{dR} / \mathrm{R}$ are easily reproduced with CKKA+TMM; a high-resolution mesh of 300 triangular oscillators was chosen to fit these data. We argue that the constrained KK method utilized here provides credibility to the resulting complex indices of refraction, since the dispersive component $\left(n\right.$, or $\left.\varepsilon_{r}\right)$ is connected directly via the KK relations to the absorptive component $\left(k\right.$, or $\left.\varepsilon_{i}\right)$, which for atomically thin films is the major contribution to the $d R / R$ measurement $[10,29]$.

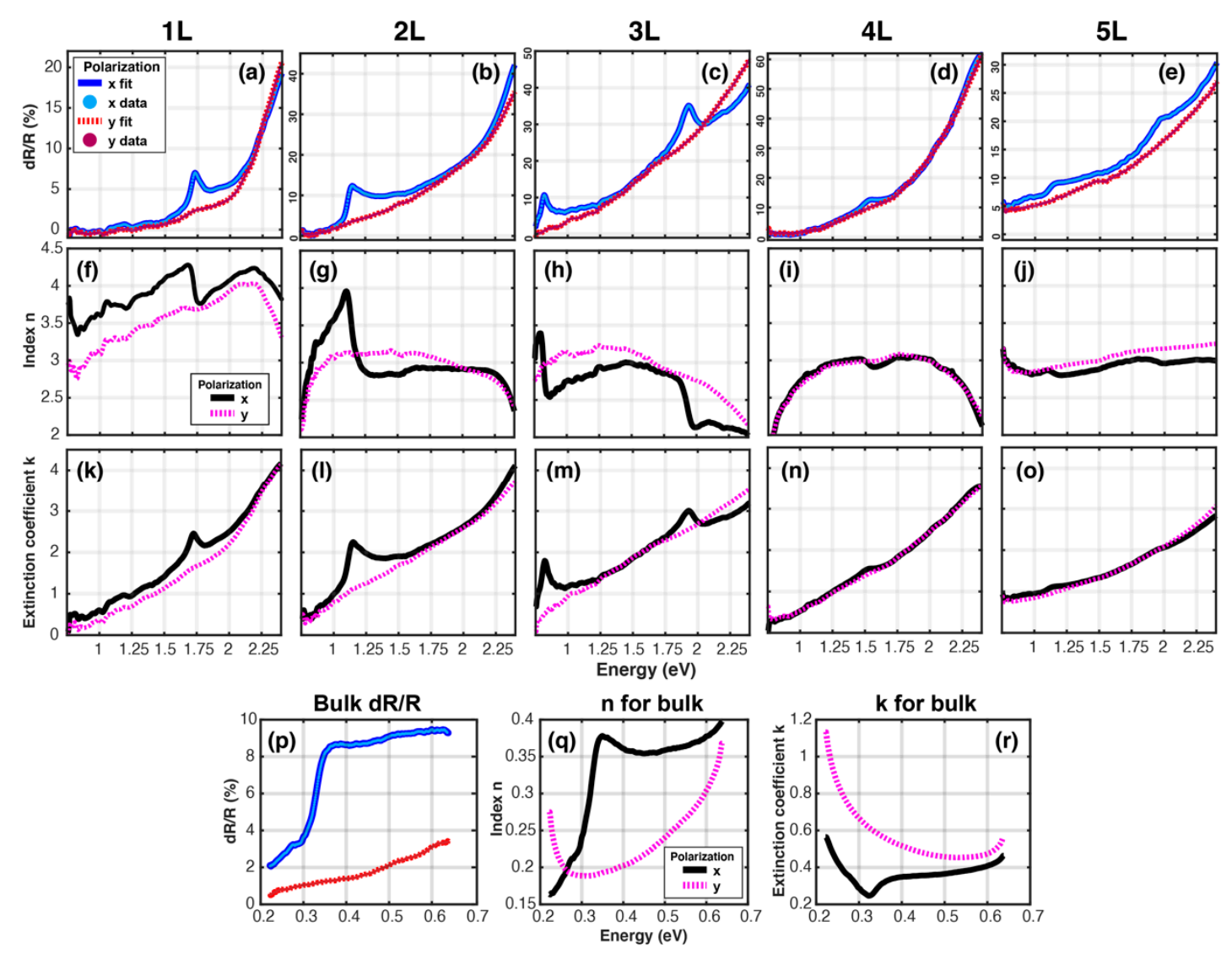

Figure 2. Complex index of refraction: results of fitting reflectance contrast $d R / R$ data for $1-5 \mathrm{~L}$ and bulk BP, taken from Li. et al. [23] using the constrained Kramers-Kronig analysis (CKKA) method. First row (a-e): polarization-selective reflectance contrast $\mathrm{dR} / \mathrm{R}\left(1-R_{\text {sample }} / R_{\text {ref }}\right)$ data, with constrained Kramers-Kronig analysis and transfer matrix method (CKKA+TMM) fits. Second row $(\mathbf{f}-\mathbf{j})$ : real index of refraction $n$ extracted using CKKA+TMM method. Third row (k-o): extinction coefficient $\mathrm{k}$ using CKKA+TMM method. Fourth row (p-r): dR/R, n, k, for bulk (100 nm) BP.

As it has been reported previously [25], the reflectance contrast data for 1-5L BP (taken at $77 \mathrm{~K}$ ) shows either one or two peaks within the experimental acquisition range of $0.75-2.4 \mathrm{eV}$, corresponding to both the intralayer exciton at lower energies and a blue-shifted exciton that arises due to interlayer effects similar to quantum wells [24]. We note that $x$ - and y-polarized excitation here corresponds to polarization aligned along the $\mathrm{AC}$ and $\mathrm{ZZ}$ directions of the puckered few-layer BP, respectively. 
This puckering effect leads to significant in-plane anisotropy, resulting in a band structure for which the effective electron mass along the AC direction is five times larger than the $\mathrm{ZZ}$ direction [24].

As revealed both in the $\mathrm{dR} / \mathrm{R}$ data, as well as in our extracted complex indices of refraction, sharp excitonic features are observed only in the $\mathrm{AC}(\mathrm{x})$ case in $\mathrm{dR} / \mathrm{R}$ and $k$, with strongly dispersive lineshapes in $n$ imposed on a relatively flat background which varies from 2-4 (Figure $2 \mathrm{k}-\mathrm{o}$ ). In the case of ZZ excitation, both the index $n$ and extinction coefficient $k$ are relatively featureless: the index is relatively flat between $2.5-3$ from $1-2.2 \mathrm{eV}$ in the cases of $2-5 \mathrm{~L}$ (Figure $2 \mathrm{~g}-\mathrm{j}$, pink dashed curve), while the extinction coefficient $k$ increases from 0 to 4 between these energy ranges (Figure 2l-o, pink dashed curve). In fact, for both $n$ and $k$, the results for ZZ excitation seem to constitute the background features in the AC case, indicating the presence of shared higher energy resonances which are isotropic. Thus, by examining the extinction coefficient $k$ directly, which corresponds to absorption, we confirm previous statements in the literature that there exists a broad and increasing background absorption in few-layer BP towards higher energy, on which the strong and narrow excitonic resonances are super-imposed.

The CKKA+TMM analysis used here is more general than the linearized models often used for the treatment of static absorption by atomically thin materials such as TMDs and few-layer BP [10,25]. Indeed, this model is used here to extract quantitatively meaningful results for the complex index of refraction, without resorting to approximations such as small optical path length for the encapsulation layer, minimal absorption in the BP layers or the neglect of the substrate reflection at the BP $\rightarrow$ $\alpha-\mathrm{Al}_{2} \mathrm{O}_{3}$ interface.

This generality allows us to also fit the $100 \mathrm{~nm}$ bulk BP sample (Figure 2, fourth row). The dR/R data are no longer easily directly correlated with the absorption coefficient $k$, due to the non-negligible thickness of bulk BP, as well as the interference effects with the hBN encapsulating layer. High quality fits of the $d R / R$ data reveal again large anisotropy resulting in birefringence of bulk BP, with a modulation of the index of refraction around $0.32 \mathrm{eV}$ in the AC case, which is not observed in the $\mathrm{ZZ}$ excitation case. For the extinction coefficient $k$, in the AC case, the absorption shows an onset around the band edge at $0.32 \mathrm{eV}$, but also shows absorption at lower energies likely due to the Drude response from free carrier excitation [24]. Surprisingly, for the ZZ excitation case, the absorption also increases steadily towards higher energies, without any dispersion modulation around the band edge.

We also note that in this energy range, the extracted index of refraction for bulk BP is below 1 . Sub-unity indices of refraction are commonly reported in noble metal materials such as Ag and Au, where there is an interplay between interband absorption and the plasmonic response [45-47] as well as in $\mathrm{Al}$ thin films at wavelengths shorter than $660 \mathrm{~nm}$ [48]. Indeed, a zero-crossing of the real part of the dielectric function $\varepsilon_{r}$ is required to satisfy the conditions for a localized surface plasmon resonance (LSPR) [49]; the plasmonic nature of BP is currently under investigation, due its novel features such as hyperbolic plasmonics arising from the large ratio between the $\mathrm{AC}$ and $\mathrm{ZZ}$ direction effective electron mass [24].

Systematics: In this section, we remark on the sensitivity of the CKKA+TMM analysis for the extraction of the complex indices of refraction to variations in parameters such as substrate and encapsulation layer refractive indices, as well as fixed fitting parameters. Previous reports involving the utilization of the CKKA method have commented on the need for incorporating information about low and high energy extremes of the dielectric functions outside of the experimental acquisition range. For instance, Li et al. [10] assumed during their studies of TMD monolayers that at high energies $(>3 \mathrm{eV})$, the bulk optical properties also contributed to the monolayer optical response; absorption resonances were included up to $30 \mathrm{eV}$. No such assumptions are made in our determination of $n, k$ for few-layer BP or the bulk BP.

However, in Figure 3, a convergence of the fitting results for 1L-x BP is shown as a function of the buffer energy that is included in the fits. More specifically, the experimental data were collected from $0.75-2.4 \mathrm{eV}$; additionally, we examined the convergence of the fit solution as a function of how far the oscillator energy mesh extended beyond these extremal values, i.e., $E_{\text {mesh }} \in\left[E_{\text {data }, i}-E_{b u f}, E_{\text {data }, f}+E_{b u f}\right]$. Although the imaginary part of the CKKA basis triangular 
dielectric function is well localized in energy, the real part exhibits long tails away from the oscillator center; thus, by extending our oscillator energy range, we can simulate how these low and high energy contributions increase the quality of fit. This buffer energy was tested from 0 to $0.3 \mathrm{eV}$; it is shown (Figure 3, center, inset) that increasing the buffer energy from 0 to $0.1 \mathrm{eV}$ reduces the sum of the residuals by nearly two orders of magnitude, with generally no enhancement beyond $0.1 \mathrm{eV}$. This convergence can be seen in the results for $n$ and $k$. However, it is noted that at energies greater than $2.4 \mathrm{eV}$ that the real index of refraction $n$ varies considerably for different buffer energies; random variations are also observed in this energy range for slight variations in the number of energy mesh points (not shown). We believe that this lack of convergence arises because of the lack of constraints on the dielectric function we have imposed on energies higher than $2.5 \mathrm{eV}$; this aspect may be potentially improved by using the higher energy absorption data for bulk BP, in a similar manner as done with TMD monolayers.

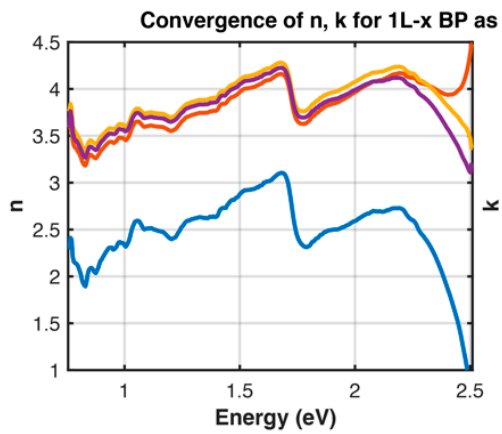

(a)

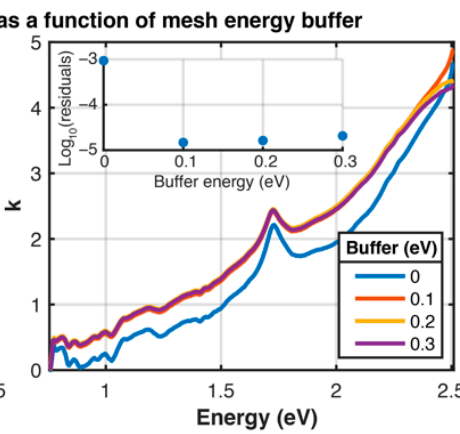

(b)

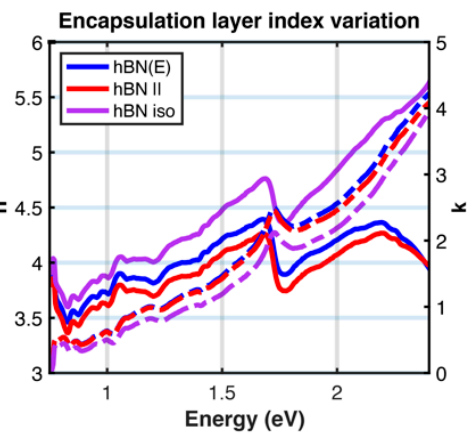

(c)

Figure 3. Systematic variation in CKKA+TMM parameters. Left (a) and center (b) plots: demonstration of convergence of CKKA+TMM fits for x-polarized excitation of 1L BP as a function of mesh energy buffer. This buffer is defined as the energy range extending beyond the experimental data acquisition range used in the CKKA+TMM fit. (a): real index of refraction $\mathrm{n}$ as a function of buffer energy in $\mathrm{eV}$ (see legend in plot on right). (b): extinction coefficient $\mathrm{k}$ as a function of buffer energy. Inset of (b): $\log _{10}$ of the residuals of the $\mathrm{dR} / \mathrm{R}$ fits as a function of buffer energy. Residuals are defined here as $\sum\left|x_{f i t, j}-x_{\text {data }, j}\right|^{2}$. (c): variation of the index of refraction for the $15 \mathrm{~nm}$ hexagonal boron nitride (hBN) encapsulating layer. $\mathrm{hBN}(\mathrm{E}), \mathrm{hBN} \|$ and $\mathrm{hBN}$ iso correspond to using the Sellmeier equation form of the index of refraction for in-plane hBN, a constant value of $n_{h B N}=2.108$ [33], and a constant value $n_{h B N}=1.86$ [34], respectively. Solid lines correspond to $\mathrm{n}$ of BP, and dashed lines correspond to $\mathrm{k}$ of BP, as indicated by solid and dashed arrows.

The dependence of the results for 1L-x BP were also studied as a function of the hBN refractive index. It is known that exfoliated hBN is birefringent, but estimates of the for in-plane and out-of-plane indices of refraction have varied significantly between reports [33-35]. For the results displayed in Figure 2, as well as Figure 3, a Sellmeier equation is used that describes the index of refraction of the in-plane index, which ranges from 2.25 to 2.1 from $400-1600 \mathrm{~nm}$ [33]. The fitting procedure was also performed for a constant index $n=2.108$, or the average of the in-plane index, and $n=1.86$, a commonly used value for isotropic BN [33-35] (Figure 3a,b). Using the constant in-plane value yields a generally lower value than the Sellmeier case, and using the isotropic value leads to much larger estimates of $n$; the variations in $k$ are not as significant (Figure 3c). We believe that the use of the functional Sellmeier equation form is appropriate; the measurement resulting in that form utilized confocal oblique incidence ellipsometry to capture both the in-plane and out-of-plane indices from 400-1600 nm [33].

Calculation of reflectance contrast of $\mathrm{BP}$ on $\mathrm{SiO}_{2} / \mathrm{Si}$ substrates: One immediately apparent use for the complex indices of refraction of BP determined in this paper using CKKA+TMM is the simulation of the optical properties of complex multi-layered devices incorporating BP. During the characterization 
and development of novel optical devices that incorporate exfoliated materials such as TMDs and BP, one step in the standard procedure for exfoliation involves the isolation of small flakes by thickness. This identification often involves measuring the reflectance contrast over the visible spectrum, i.e., examining a microscope image by color contrast (by eye or CCD) [50]. Thus, maximizing the color contrast between the active layer (TMD or BP) and the substrate is essential for this exfoliation procedure. If the reflectance contrast curves can be predicted ahead of time given knowledge of the complex indices of refraction of the TMD/BP and the substrate, the thickness of the active layer can be determined with certainty.

In TMD systems, one common substrate employed for high color contrast is $90-120 \mathrm{~nm}$ of $\mathrm{SiO}_{2}$ on a Si substrate [28]. Here, we employed the TMM to calculate the expected reflectance contrast of one to three layers of BP on that same substrate, using the complex dielectric constants determined in this article via CKKA+TMM. Thus, we have provided a useful reference for optical physicists attempting to incorporate BP in their devices. The results of the calculation are shown in Figure 4 . It becomes immediately clear that this standard substrate does not provide high reflectance contrast, at least not as high as using sapphire as a substrate (Figure 2). This lack of reflectance contrast is due to the relative similarities of the real parts of the index of refraction for Si and 1-3 L BP, with n $\sim 3-4$ from 0.8 to $2.5 \mathrm{eV}$ (Figure 2). However, the contrast between the $\mathrm{x}$ - and $\mathrm{y}$-polarized excitation reflectance contrast allows one to clearly locate sharp features in the optical spectrum, which can then be used for layer thickness determination. For easy thickness determination, we suggest utilizing either sapphire as a substrate, or polarization-dependent reflectance contrast.
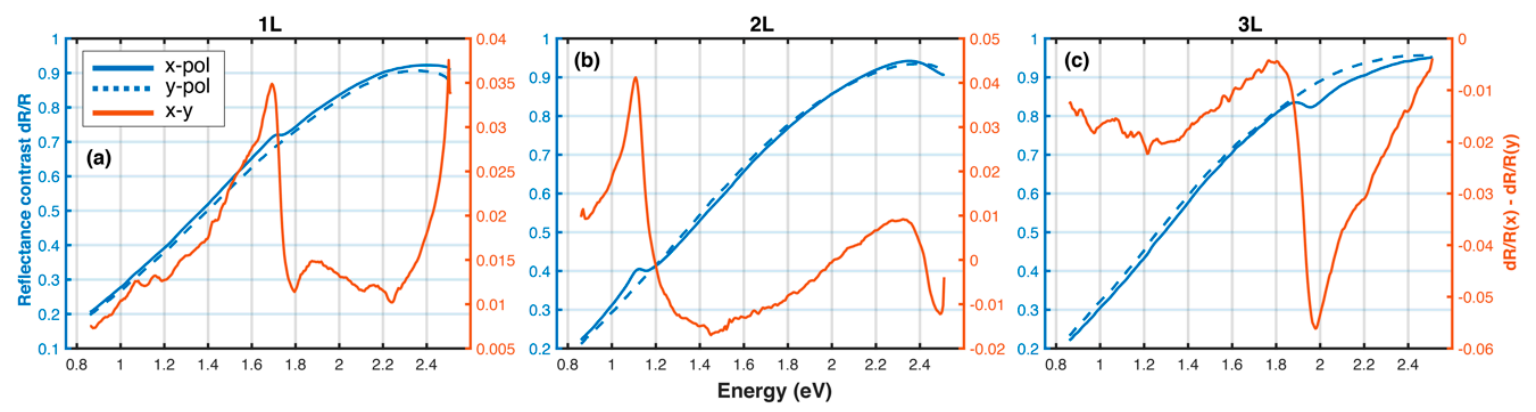

Figure 4. Reflectance contrast (dR/R) calculated using n,k displayed in Figure 2 between (1L (a), 2L (b), and 3L (c), $\mathrm{BP}$ on $90 \mathrm{~nm} \mathrm{SiO} / \mathrm{Si}$ ) and $90 \mathrm{~nm} \mathrm{SiO} / 2 \mathrm{Si}$. Solid blue, dashed blue and solid orange curves correspond to $\mathrm{dR} / \mathrm{R}$ for the $\mathrm{x}$-polarization, $\mathrm{y}$-polarization excitation conditions and the difference between the two, respectively.

Discussion of study limitations, and comparisons to the literature: Some of the limitations of this study have been discussed above, and we re-iterate in more detail here. First, as discussed in detail by Li et al. [10] and Kuzmenko [37], both the standard Kramers-Kronig method and the CKKA method utilized in this article are sensitive to the optical response of the system outside of the experimental acquisition range. The standard KK approach requires that the absorptive optical response is localized over an energy range smaller than the experimental energy range; while the CKKA method does not explicitly require this, we showed in the Systematics section of this article that the extracted complex index of refraction has high uncertainty at the edges of the data acquisition range. The se uncertainties would be greatly reduced by incorporating both higher and lower energy data that could be acquired via electron energy-loss measurements and UV-XUV absorption measurements [41], as well as long wavelength FTIR spectroscopy. We note that it is non-trivial to incorporate the results of those higher/lower energy experiments into CKKA+TMM, since those experiments span over highly disparate energy ranges and apply to the bulk crystal black phosphorous, but point the reader towards referenced literature for future studies [51-54].

Second, both the KK and CKKA methods require precise characterization of the thicknesses of the substrate layers with nanometer precision, and knowledge of the indices of refraction for those layers. 
In this article, the hBN layer thickness has been taken as approximately $15 \mathrm{~nm}$, as given by Li et al. [25]. We suggest that a more consistent approach would involve multiple reflectance contrast measurements of BP with varying hBN encapsulation layer thicknesses, with thicknesses of all layers characterized by atomic force microscopy. The same concern applies to the extraction of the complex index of the bulk $\mathrm{BP}$ described in this article; this thickness was taken to be equal to approximately $100 \mathrm{~nm}$, as given by Li et al. [25], but the uncertainty on this thickness is unknown to us. Additionally, we are uncertain of the crystallinity of the hBN encapsulation layer, as discussed in the Systematics section of this article, but have provided systematic variations over the possible cases.

In the context of the literature regarding the optical characterization of TMDs and few-layer BP, and more generally exfoliated materials, this article takes a similar approach to that of Li et al. [10], in which the complex dielectric functions of monolayer TMDs were determined via CKKA+TMM. The analysis provided here clearly separates the dispersive (real) and absorptive (imaginary) parts of the optical dielectric constants of few-layer and bulk BP, which is not obvious in reflectance contrast data.

\section{Conclusions}

For the estimate of the index of the refraction of black phosphorus, we have demonstrated that the constrained KK analysis combined with a general transfer matrix method (CKKA+TMM) that takes into account finite layer optical path lengths can be used to extract the entire complex index of the refraction of 1-5 L BP and bulk BP from reflectance contrast data. It is confirmed that the excitonic peaks correspond to peaks in the extinction coefficient $k$, and that a significant absorptive background that increases towards higher energies exists. In contrast, the real index of refraction is relatively flat with superimposed dispersive features located at the excitonic resonances. The determination of the full complex index of refraction $\tilde{n}$ of few-layer BP is significant: these results may be incorporated into the development of novel optical devices such as responsive photonic crystals and distributed Bragg reflectors (DBRs) [55-58], plasmonic nanostructures [59] and on-chip waveguides, all of which require knowledge of both the dispersive and absorptive optical properties. We calculated the reflectance contrast of $1-3 \mathrm{~L} \mathrm{BP}$ on a $\mathrm{SiO}_{2} / \mathrm{Si}$ substrate, providing a useful reference for physicists working to determine thicknesses of exfoliated BP. We suggest further improvements to this extractive method that would involve the experimental acquisition of both reflectance and transmission contrast simultaneously, as well as constraining the low and high energy ranges in the CKKA+TMM analysis with optical properties deduced from FTIR and UV absorption.

Author Contributions: Conceptualization, A.M.R., G.M.P., S.D.C., F.S. and E.C.; methodology, validation and formal analysis, A.M.R., F.S. and E.C.; writing-original draft preparation, A.M.R. and F.S.; writing-review and editing, A.M.R., G.M.P., S.D.C., F.S. and E.C. All authors have read and agreed to the published version of the manuscript.

Funding: This project has received funding from the European Research Council (ERC) under the European Union's Horizon 2020 research and innovation programme (grant agreement No. [816313]). E.C. acknowledges financial support from MIUR PRIN aSTAR, Grant No. 2017RKWTMY and from the European Research Council through the MSCA-ITN SMART-X (GA 860553). G.M.P. acknowledges the financial support from Fondazione Cariplo, grant no. 2018-0979. S.D.C. acknowledges financial support from MIUR through the PRIN 2017 Programme (Prot. 20172H2SC4).

Conflicts of Interest: The authors declare no conflict of interest.

\section{References}

1. Mak, K.F.; Lee, C.; Hone, J.; Shan, J.; Heinz, T.F. Atomically Thin MoS2: A New Direct-Gap Semiconductor. Phys. Rev. Lett. 2010, 105, 136805. [CrossRef] [PubMed]

2. Zeng, H.; Dai, J.; Yao, W.; Xiao, D.; Cui, X. Valley polarization in MoS2 monolayers by optical pumping. Nat. Nanotechnol. 2012, 7, 490-493. [CrossRef] [PubMed] 
3. Huang, L.; McCormick, T.M.; Ochi, M.; Zhao, Z.; Suzuki, M.-T.; Arita, R.; Wu, Y.; Mou, D.; Cao, H.; Yan, J.; et al. Spectroscopic evidence for a type II Weyl semimetallic state in MoTe2. Nat. Mater. 2016, 15, 1155-1160. [CrossRef] [PubMed]

4. Tao, L.; Cinquanta, E.; Chiappe, D.; Grazianetti, C.; Fanciulli, M.; Dubey, M.; Molle, A.; Akinwande, D. Silicene field-effect transistors operating at room temperature. Nat. Nanotechnol. 2015, 10, 227-231. [CrossRef]

5. Wood, J.D.; Wells, S.A.; Jariwala, D.; Chen, K.-S.; Cho, E.; Sangwan, V.K.; Liu, X.; Lauhon, L.J.; Marks, T.J.; Hersam, M.C. Effective Passivation of Exfoliated Black Phosphorus Transistors against Ambient Degradation. Nano Lett. 2014, 14, 6964-6970. [CrossRef]

6. Churchill, H.O.H.; Jarillo-Herrero, P. Phosphorus joins the family. Nat. Nanotechnol. 2014, 9, 330-331. [CrossRef]

7. Shen, C.-C.; Hsu, Y.-T.; Li, L.-J.; Liu, H.-L. Charge Dynamics and Electronic Structures of Monolayer MoS2 Films Grown by Chemical Vapor Deposition. Appl. Phys. Express 2013, 6, 125801. [CrossRef]

8. Yim, C.; O’Brien, M.; McEvoy, N.; Winters, S.; Mirza, I.; Lunney, J.G.; Duesberg, G.S. Investigation of the optical properties of MoS2 thin films using spectroscopic ellipsometry. Appl. Phys. Lett. 2014, 104, 103114. [CrossRef]

9. Li, W.; Birdwell, A.G.; Amani, M.; Burke, R.A.; Ling, X.; Lee, Y.-H.; Liang, X.; Peng, L.; Richter, C.A.; Kong, J.; et al. Broadband optical properties of large-area monolayer CVD molybdenum disulfide. Phys. Rev. B 2014, 90, 195434. [CrossRef]

10. Li, Y.; Chernikov, A.; Zhang, X.; Rigosi, A.; Hill, H.M.; Van Der Zande, A.M.; Chenet, D.A.; Shih, E.M.; Hone, J.; Heinz, T.F. Measurement of the optical dielectric function of monolayer transition-metal dichalcogenides: MoS2, MoSe2, WS2, and WSe2. Phys. Rev. B 2014, 90, 1-6. [CrossRef]

11. Hsu, C.; Frisenda, R.; Schmidt, R.; Arora, A.; Vasconcellos, S.M.; Bratschitsch, R.; der Zant, H.S.J.; Castellanos-Gomez, A. Thickness-Dependent Refractive Index of 1L, 2L, and 3L MoS2, MoSe2, WS2, and WSe2. Adv. Opt. Mater. 2019, 7, 1900239. [CrossRef]

12. Ermolaev, G.A.; Stebunov, Y.V.; Vyshnevyy, A.A.; Tatarkin, D.E.; Yakubovsky, D.I.; Novikov, S.M.; Baranov, D.G.; Shegai, T.; Nikitin, A.Y.; Arsenin, A.V.; et al. Broadband optical properties of monolayer and bulk MoS 2. npj 2D Mater. Appl. 2020, 4, 1-6. [CrossRef]

13. Ermolaev, G.A.; Yakubovsky, D.I.; Stebunov, Y.V.; Arsenin, A.V.; Volkov, V.S. Spectral ellipsometry of monolayer transition metal dichalcogenides: Analysis of excitonic peaks in dispersion. J. Vac. Sci. Technol. B 2019, 38, 14002. [CrossRef]

14. Li, H.; Contryman, A.W.; Qian, X.; Ardakani, S.M.; Gong, Y.; Wang, X.; Weisse, J.M.; Lee, C.H.; Zhao, J.; Ajayan, P.M.; et al. Optoelectronic crystal of artificial atoms in strain-textured molybdenum disulphide. Nat. Commun. 2015, 6, 7381. [CrossRef] [PubMed]

15. Cordovilla Leon, D.F.; Li, Z.; Jang, S.W.; Cheng, C.-H.; Deotare, P.B. Exciton transport in strained monolayer WSe2. Appl. Phys. Lett. 2018, 113, 252101. [CrossRef]

16. Robert, C.; Amand, T.; Cadiz, F.; Lagarde, D.; Courtade, E.; Manca, M.; Taniguchi, T.; Watanabe, K.; Urbaszek, B.; Marie, X. Fine structure and lifetime of dark excitons in transition metal dichalcogenide monolayers. Phys. Rev. B 2017, 96, 155423. [CrossRef]

17. Zhou, Y.; Scuri, G.; Wild, D.S.; High, A.A.; Dibos, A.; Jauregui, L.A.; Shu, C.; De Greve, K.; Pistunova, K.; Joe, A.Y.; et al. Probing dark excitons in atomically thin semiconductors via near-field coupling to surface plasmon polaritons. Nat. Nanotechnol. 2017, 12, 856-860. [CrossRef]

18. Klein, M.; Badada, B.H.; Binder, R.; Alfrey, A.; McKie, M.; Koehler, M.R.; Mandrus, D.G.; Taniguchi, T.; Watanabe, K.; LeRoy, B.J.; et al. 2D semiconductor nonlinear plasmonic modulators. Nat. Commun. 2019, 10, 3264. [CrossRef]

19. Camellini, A.; Mennucci, C.; Cinquanta, E.; Martella, C.; Mazzanti, A.; Lamperti, A.; Molle, A.; de Mongeot, F.B.; Della Valle, G.; Zavelani-Rossi, M. Ultrafast Anisotropic Exciton Dynamics in Nanopatterned MoS2 Sheets. ACS Photonics 2018, 5, 3363-3371. [CrossRef]

20. Castellanos-Gomez, A.; Roldán, R.; Cappelluti, E.; Buscema, M.; Guinea, F.; van der Zant, H.S.J.; Steele, G.A. Local Strain Engineering in Atomically Thin MoS2. Nano Lett. 2013, 13, 5361-5366. [CrossRef]

21. Quereda, J.; San-Jose, P.; Parente, V.; Vaquero-Garzon, L.; Molina-Mendoza, A.J.; Agraït, N.; Rubio-Bollinger, G.; Guinea, F.; Roldán, R.; Castellanos-Gomez, A. Strong Modulation of Optical Properties in Black Phosphorus through Strain-Engineered Rippling. Nano Lett. 2016, 16, 2931-2937. [CrossRef] [PubMed] 
22. Slobodeniuk, A.O.; Basko, D.M. Spin-flip processes and radiative decay of dark intravalley excitons in transition metal dichalcogenide monolayers. 2D Mater. 2016, 3, 035009. [CrossRef]

23. Molas, M.R.; Nogajewski, K.; Slobodeniuk, A.O.; Binder, J.; Bartos, M.; Potemski, M. The optical response of monolayer, few-layer and bulk tungsten disulfide. Nanoscale 2017, 9, 13128-13141. [CrossRef] [PubMed]

24. Wang, C.; Zhang, G.; Huang, S.; Xie, Y.; Yan, H. The Optical Properties and Plasmonics of Anisotropic 2D Materials. Adv. Opt. Mater. 2020, 8, 1-22. [CrossRef]

25. Li, L.; Kim, J.; Jin, C.; Ye, G.J.; Qiu, D.Y.; Da Jornada, F.H.; Shi, Z.; Chen, L.; Zhang, Z.; Yang, F.; et al. Direct observation of the layer-dependent electronic structure in phosphorene. Nat. Nanotechnol. 2017, 12, 21-25. [CrossRef] [PubMed]

26. He, Y.M.; Clark, G.; Schaibley, J.R.; He, Y.; Chen, M.C.; Wei, Y.J.; Ding, X.; Zhang, Q.; Yao, W.; Xu, X.; et al. Single quantum emitters in monolayer semiconductors. Nat. Nanotechnol. 2015, 10, 497-502. [CrossRef]

27. Mao, N.; Tang, J.; Xie, L.; Wu, J.; Han, B.; Lin, J.; Deng, S.; Ji, W.; Xu, H.; Liu, K.; et al. Optical Anisotropy of Black Phosphorus in the Visible Regime. J. Am. Chem. Soc. 2016, 138, 300-305. [CrossRef]

28. Castellanos-Gomez, A.; Agrat, N.; Rubio-Bollinger, G. Optical identification of atomically thin dichalcogenide crystals. Appl. Phys. Lett. 2010, 96, 213116. [CrossRef]

29. Li, Y.; Heinz, T.F. Two-dimensional models for the optical response of thin films. 2D Mater. 2018, 5, 025021. [CrossRef]

30. Mak, K.F.; Sfeir, M.Y.; Wu, Y.; Lui, C.H.; Misewich, J.A.; Heinz, T.F. Measurement of the Optical Conductivity of Graphene. Phys. Rev. Lett. 2008, 101, 196405. [CrossRef]

31. Liu, H.L.; Shen, C.C.; Su, S.H.; Hsu, C.L.; Li, M.Y.; Li, L.J. Optical properties of monolayer transition metal dichalcogenides probed by spectroscopic ellipsometry. Appl. Phys. Lett. 2014, 105, 201905. [CrossRef]

32. Park, J.W.; So, H.S.; Kim, S.; Choi, S.H.; Lee, H.; Lee, J.; Lee, C.; Kim, Y. Optical properties of large-area ultrathin MoS2 films: Evolution from a single layer to multilayers. J. Appl. Phys. 2014, 116, 183509. [CrossRef]

33. Rah, Y.; Jin, Y.; Kim, S.; Yu, K. Optical analysis of the refractive index and birefringence of hexagonal boron nitride from the visible to near-infrared. Opt. Lett. 2019, 44, 3797. [CrossRef] [PubMed]

34. Schubert, M.; Rheinländer, B.; Franke, E.; Neumann, H.; Hahn, J.; Röder, M.; Richter, F. Anisotropy of boron nitride thin-film reflectivity spectra by generalized ellipsometry. Appl. Phys. Lett. 1997, 70, 1819-1821. [CrossRef]

35. Lee, S.Y.; Jeong, T.Y.; Jung, S.; Yee, K.J. Refractive Index Dispersion of Hexagonal Boron Nitride in the Visible and Near-Infrared. Phys. Status Solidi Basic Res. 2019, 256, 1-6. [CrossRef]

36. DeFranzo, A.C.; Pazol, B.G. Index of refraction measurement on sapphire at low temperatures and visible wavelengths. Appl. Opt. 1993, 32, 2224. [CrossRef]

37. Kuzmenko, A.B. Kramers- Kronig constrained variational analysis of optical spectra. Rev. Sci. Instrum. 2005, 76, 1-9. [CrossRef]

38. Mayerhöfer, T.G.; Popp, J. Improving Poor Man's Kramers-Kronig analysis and Kramers-Kronig constrained variational analysis. Spectrochim. Acta Part A Mol. Biomol. Spectrosc. 2019, 213, 391-396. [CrossRef]

39. Jackson, J.D. Classical Electrodynamics, 3rd ed.; Wiley: Hoboken, NY, USA, 2009; ISBN 978-0-471-30932-1.

40. Beal, A.R.; Liang, W.Y.; Hughes, H.P. Kramers-Kronig analysis of the reflectivity spectra of 3R-WS2 and 2H-WSe2. J. Phys. C Solid State Phys. 1976, 9, 2449-2457. [CrossRef]

41. Beal, A.R.; Hughes, H.P. Kramers- Kronig analysis of the reflectivity spectra of 2H-MoS2, 2H-MoSe2 and 2H-MoTe2. J. Phys. C Solid State Phys. 1979, 12, 881-890. [CrossRef]

42. Li, H.; Zhang, Q.; Yap, C.C.R.; Tay, B.K.; Edwin, T.H.T.; Olivier, A.; Baillargeat, D. From bulk to monolayer MoS 2: Evolution of Raman scattering. Adv. Funct. Mater. 2012, 22, 1385-1390. [CrossRef]

43. Hecht, E. Optics, 3rd ed.; Addison-Wesley: Boston, MA, USA, 1998; ISBN 0201838877.

44. Ankit Rohatgi. WebPlotDigitizer; vesion 4.4; Ankit Rohatgi: Pacifica, CA, USA, 2020.

45. Yang, J.; Xu, R.; Pei, J.; Myint, Y.W.; Wang, F.; Wang, Z.; Zhang, S.; Yu, Z.; Lu, Y. Optical tuning of exciton and trion emissions in monolayer phosphorene. Light Sci. Appl. 2015, 4, e312. [CrossRef]

46. Johnson, P.B.; Christy, R.W. Optical Constants of the Noble Metals. Phys. Rev. B 1972, 6, 4370-4379. [CrossRef]

47. Ehrenreich, H.; Philipp, H.R. Optical Properties of Ag and Cu. Phys. Rev. 1962, 128, 1622-1629. [CrossRef]

48. Cheng, F.; Su, P.-H.; Choi, J.; Gwo, S.; Li, X.; Shih, C.-K. Epitaxial Growth of Atomically Smooth Aluminum on Silicon and Its Intrinsic Optical Properties. ACS Nano 2016, 10, 9852-9860. [CrossRef]

49. Maier, S.A. Plasmonics Fundamentals and Applications; Springer: New York, NY, USA, 2007; ISBN 9780387331508. 
50. Niu, Y.; Gonzalez-Abad, S.; Frisenda, R.; Marauhn, P.; Drüppel, M.; Gant, P.; Schmidt, R.; Taghavi, N.; Barcons, D.; Molina-Mendoza, A.; et al. Thickness- Dependent Differential Reflectance Spectra of Monolayer and Few-Layer MoS2, MoSe2, WS2 and WSe2. Nanomaterials 2018, 8, 725. [CrossRef]

51. Harada, Y.; Murano, K.; Shirotani, I.; Takahashi, T.; Maruyama, Y. Electronic structure of black phosphorus studied by X-ray photoelectron spectroscopy. Solid State Commun. 1982, 44, 877-879. [CrossRef]

52. Ikezawa, M.; Kondo, Y.; Shirotani, I. Infrared Optical Absorption Due to One and Two Phonon Processes in Black Phosphorus. J. Phys. Soc. Japan 1983, 52, 1518-1520. [CrossRef]

53. Takahashi, T.; Shirotani, K.; Suzuki, S.; Sagawa, T. Band structure of black phosphorus studied by angle-resolved ultraviolet photoemission spectroscopy. Solid State Commun. 1983, 45, 945-948. [CrossRef]

54. Taniguchi, M.; Suga, S.; Seki, M.; Sakamoto, H.; Kanzaki, H.; Akahama, Y.; Terada, S.; Endo, S.; Narita, S. Valence band and core-level photoemission spectra of black phosphorus single crystals. Solid State Commun. 1983, 45, 59-61. [CrossRef]

55. Bonifacio, L.D.; Lotsch, B.V.; Puzzo, D.P.; Scotognella, F.; Ozin, G.A. Stacking the Nanochemistry Deck: Structural and Compositional Diversity in One-Dimensional Photonic Crystals. Adv. Mater. 2009, 21, 1641-1646. [CrossRef]

56. Paternò, G.M.; Manfredi, G.; Scotognella, F.; Lanzani, G. Distributed Bragg reflectors for the colorimetric detection of bacterial contaminants and pollutants for food quality control. APL Photonics 2020, 5, 080901. [CrossRef]

57. Moscardi, L.; Paternò, G.M.; Chiasera, A.; Sorrentino, R.; Marangi, F.; Kriegel, I.; Lanzani, G.; Scotognella, F. Electro-responsivity in electrolyte-free and solution processed Bragg stacks. J. Mater. Chem. C 2020, 8, 13019-13024. [CrossRef]

58. Normani, S.; Dalla Vedova, N.; Lanzani, G.; Scotognella, F.; Paternò, G.M. Design of 1D photonic crystals for colorimetric and ratiometric refractive index sensing. Opt. Mater. X 2020, 8, 100058. [CrossRef]

59. Ferry, V.E.; Munday, J.N.; Atwater, H.A. Design considerations for plasmonic photovoltaics. Adv. Mater. 2010, 22, 4794-4808. [CrossRef] [PubMed]

Publisher's Note: MDPI stays neutral with regard to jurisdictional claims in published maps and institutional affiliations.

(C) 2020 by the authors. Licensee MDPI, Basel, Switzerland. This article is an open access article distributed under the terms and conditions of the Creative Commons Attribution (CC BY) license (http://creativecommons.org/licenses/by/4.0/). 